\title{
Tuning the Mechanical Properties of Nanoporous Hydrogel Particles via Polymer Crosslinking
}

\author{
James P. Best, Jiwei Cui, Markus Müllner, and Frank Caruso* \\ Department of Chemical and Biomolecular Engineering, The University of Melbourne, Parkville, Victoria 3010, Aus- \\ tralia
}

Email: fcaruso@unimelb.edu.au

Supporting Information

\begin{abstract}
Soft hydrogel particles with tunable mechanical properties are promising for next-generation therapeutic applications. This is due to the increasingly proven role that physicochemical properties play in particulate-based delivery vectors, both in vitro and in vivo. The ability to understand and quantify the mechanical properties of such systems is therefore essential to optimize function and performance. We report control over the mechanical properties of poly(methacrylic acid) (PMA) hydrogel particles based on a mesoporous silica templating method. The mechanical properties of the obtained particles can be finely tuned through variation of the cross-linker concentration, which is hereby quantified using a crosslinking polymer with a fluorescent tag. We demonstrate that the mechanical properties of the particles can be elucidated using an atomic force microscopy (AFM) force spectroscopy method, which additionally allows for the study of hydrogel material properties at the nanoscale through high-resolution force mapping. The Young's modulus and stiffness of the particles was tuned between 0.04 to $2.53 \mathrm{MPa}$ and 1.6 to $28.4 \mathrm{mN} \mathrm{m}^{-1}$, respectively, through control over the cross-linker concentration. The relationship between concentration of cross-linker added and amount of adsorbed polymer were observed to follow a Langmuir isotherm, and this relationship was found to correlate linearly with the particle mechanical properties.
\end{abstract}

\section{INTRODUCTION}

Nanostructured particulate materials are expected to revolutionize diagnostics and the delivery of therapeutics. ${ }^{1-4}$ Such finely engineered systems have a number of advantages, including; precise targeting of the therapeutic, improved solubility and circulation half-life, reduced immunogenicity, and improved therapeutic index. ${ }^{4}$ Submicron-sized hydrogel particles with tunable physicochemical properties have demonstrated in vitro vaccine delivery, ${ }^{5}$ nitric oxide inhibition, ${ }^{6}$ and effective doxorubicin delivery to colorectal cancer cells. ${ }^{7}$ While research into such treatments has progressed considerably, the efficiency of therapeutic vectors for intracellular delivery is often limited by their interactions with cell membranes. ${ }^{8}$ To optimize particulate delivery for biomedical applications, precise and tunable control over the surface chemistry, geometry and mechanics has therefore been outlinded as an effective approach for generating next-generation clinical models.4,9,10

Experimental studies highlight the influence of hydrogel mechanical properites on fundamental circulatory or cellular responses during therapy. ${ }^{10}$ Stiff particles have been shown to be more efficiently cleared from the body via the immune system than softer elastic counterparts.,41,12 For example, Discher and coworkers found that flexible filamentous micelles align in capillary flow streams and evade phagocytic clearance. ${ }^{12}$ This observation was found to be a result of the mechanics of the long micelle, as subsequent crosslinking (and resultant stiffness increase) led to rapid clearance. Additionally, studies have shown that the mechanical propeties of hydrogel microparticles have an influence on cellular uptake mechanisms and kinetics. ${ }^{13,14}$ Banquy et al. demonstrated that although more rigid acrylamide particles were favorable for internalization into RAW 264.7 murine macrophages, particles with an intermediate elastic modulus exhibited rapid uptake kinetics as they were able to utilize multiple internalization mechanisms. ${ }^{14}$ Such investigations highlight the importance of fine-tuning elastic properties for mediating cellular response.

The elastic properties of single submicron-sized hydrogel particles are generally characterized using atomic force microscopy (AFM) force spectroscopy. ${ }^{5,14,15}$ This allows for the material compliance $\left(k^{-1}\right)$ and Young's 
modulus $\left(E_{Y}\right)$ to be elucidated using controlled intendation measurements. This is a useful technique for monitoring subtle changes in particle mechanical properties arising from different crosslinking degrees, ${ }^{14}$ buffer $\mathrm{pH},{ }^{16}$ or other external triggers. ${ }^{15}$ Von Klitzing and coworkers, using AFM, recently demonstrated a reversible temperature-dependency for the $E_{Y}$ of $\operatorname{poly}(N-$ isopropylacrylamide-co-acrylic acid) microparticles. ${ }^{15}$ Due to the wide range of AFM cantilever spring constants available, it is relatively straightforward to match material and cantilever compliance, allowing for the measurement of extremely soft materials. Using AFM force spectroscopy, studies have previously reported on the relationship between the mechanical properties of nanostructured systems, and the extent of chemical crosslinking.,517-19 Generally, a limiting relationship has been found, where film properties plateau with increasing crosslinking degree. This was demonstrated for planar polyelectrolye films by Picart and coworkers, who observed such a relationship dependence between mechanical properties and the concentration of carbodiimide cross-linker introduced. ${ }^{20}$ This was likely due to the linear relationship found between film mechanics and cross-linker density, quantified using Fourier-transform infrared spectroscopy.

We recently reported a facile and robust approach for the preparation of polymer replica particles via mesoporous silica (MS) templating..$^{21}$ This approach has been shown to be versatile in terms of polymer type and crosslinking strategy, and can also be used to tailor the composition, morphology, and porosity of the nanostructures, and their responsiveness to biological stimuli., ${ }^{521}$ Different therapeutics have been incorperated into such polymer replica particles for drug and gene delivery. ${ }^{22}$ In this article, we sought to investigate the influence of crosslinking density on the mechanical properties of submicron-sized hydrogel particles. A bulk hydrogel was obtained via polymer infiltration into MS template particles together with varying amounts of crosslinking polymer, followed by template removal. Through control over the amount of added cross-linker, the obtained nanoporous polymer replica particles exhibit tunable crosslinking density and mechanics. Fluorescent tagging of the cross-linker allowed for quantificaion of the cross-linker amount that had been incorporated into the particle. This provides a versatile approach for determining the amount of cross-linker in hydrogel microparticle systems, and allows correlation of this parameter to the tunable mechanical properties elucidated from AFM force spectroscopy measurements. To our knowledge, this represents the first quantificationbased analysis of the mechanisms for mechanical strengthening of nanostructured hydrogel particles. Such efforts are crucial for the development of hydrogel therapeutic delivery vectors with tailored mechanical properties. Herein, this is achieved through the novel coupling of fluorescence spectrophotometry with AFM for the interrogation of sub-micron-sized PMA $\mathrm{ASH}_{\mathrm{SH}}$ hydrogels.

\section{EXPERIMENTAL METHODS}

Materials. The $\mathrm{pH}$ of all solutions was measured with a Mettler-Toledo MP220 $\mathrm{pH}$ meter. High purity (Milli-Q) water with a resistivity of $>18.2 \mathrm{M} \Omega \mathrm{cm}$ was obtained from an inline Millipore RiOs/Origin water purification system. Dialysis tubing $(68035-35 \mathrm{FT})$ with a molecular weight cutoff (MWCO) of 3500 Da was obtained from Thermo Scientific. Poly(methacrylic acid, sodium salt) $\left(M_{\mathrm{W}}=15\right.$ $\mathrm{kDa}$ ) was purchased from Polysciences. 1-Ethyl-3-(3dimethylaminopropyl) carbodiimide (EDC), dithiothreitol (DTT), ammonia (28 - 30\%), (3aminopropyl)triethoxysilane (APTES), polyethyleneimine (PEI, 25 ooo $\mathrm{g} \mathrm{mol}^{-1}$ ), phosphate buffered saline (PBS), 3( $N$-morpholino)propanesulfonic acid (MOPS), 2- $(N$ morpholino)ethanesulfonic acid (MES), sodium acetate $(\mathrm{NaOAc})$, and hydrofluoric acid (HF) were obtained from Sigma-Aldrich. Cadaverine-functionalized Alexa Fluor 488 dye $\left(\mathrm{AF}_{4} 88\right)$ was purchased from Invitrogen (Australia). Pyridine dithioethylamine hydrochloride $(\mathrm{PDA} \cdot \mathrm{HCl}$ ) was purchased from Speed Chemicals (Shanghai). All chemicals were used as received. Nuclear magnetic resonance (NMR) spectra were recorded in $\mathrm{D}_{2} \mathrm{O}$ using a 400 $\mathrm{MHz}$ Varian INOVA system at $25^{\circ} \mathrm{C}$.

Polymer Preparation. PMA sodium salt (100 $\mathrm{mg}$ ) was dissolved in a filtered $(0.2 \mu \mathrm{m})$ and deoxygenated $\left(\mathrm{N}_{2}(\mathrm{~g})\right.$ stream, $15 \mathrm{~min}$ ) PBS solution ( $25 \mathrm{~mL}, 100 \mathrm{mM}, \mathrm{pH} 7.4$ ). $\mathrm{PDA} \cdot \mathrm{HCl}$ was added in a stoichiometric excess of 1.75 equivalents of the target PDA-functionalization percentage ( $15 \mathrm{~mol} \%)$ and EDC in a stoichiometric excess of 2.90 equivalents under stirring at $24{ }^{\circ} \mathrm{C}$. The resulting solution was stirred at $24{ }^{\circ} \mathrm{C}$ for $18 \mathrm{~h}$ under a $\mathrm{N}_{2}(\mathrm{~g})$ atmosphere, after which the solution was dialyzed against Milli-Q water over a period of 5 days, with frequent exchange of the dialysis solution. The resulting purified solution was freeze-dried to give PMA-PDA as a white solid.

Preparation of PMAsH Cross-Linker. Aliquots (30 mg) of purified PMA-PDA were reacted with the fluorescent label, $\mathrm{AF}_{488}$, using carbodiimide linking chemistry. The aliquot of PMA-PDA was first dissolved in $3 \mathrm{~mL}$ of PBS (100 mM, pH 8.o), $10 \mathrm{mg}$ EDC was added, and the mixture was stirred for $10 \mathrm{~min}$. To this solution, $30 \mu \mathrm{L}$ of AF488 (1 $\mathrm{mg} \mathrm{mL}^{-1}$ in DMSO) was added, and the reaction mixture was stirred overnight, while protected from light. The resulting fluorescently labeled polymer was dialyzed against Milli-Q water for $48 \mathrm{~h}$, with frequent exchange of the dialysis solution. The purified solution was freezedried to give the product as a light-yellow solid. Labeled PMA-PDA was stored at $4{ }^{\circ} \mathrm{C}$. To convert the $\mathrm{AF}_{488}$ labeled PMA-PDA to a thiol-functionalized PMA (PMA $\left.{ }_{\mathrm{SH}}\right)$ reaction solution $\left(2 \mathrm{mg} \mathrm{mL}^{-1}\right), 1 \mathrm{mg}$ of labeled PMA-PDA was dissolved into $10 \mu \mathrm{L}$ of $0.5 \mathrm{M}$ DTT (MOPS, $20 \mathrm{mM}, \mathrm{pH}$ 8.o) and $10 \mu \mathrm{L}$ of Milli-Q water, and incubated for at least $30 \mathrm{~min}$ at $37{ }^{\circ} \mathrm{C}$ with constant agitation to cleave the 2mercaptopyridine moieties to leave reactive free thiol 
groups. PMA $A_{S H}$ was then diluted to $100 \mu \mathrm{L}$, and purified via size exclusion chromatography (SEC) using a NAP-5 desalting column equilibrated using NaOAc buffer $(\mathrm{pH}$ 5.0). The purified PMA $\mathrm{PH}_{S \mathrm{H}}$ was then collected into $500 \mu \mathrm{L}$ of $\mathrm{NaOAc}$ buffer to give the $2 \mathrm{mg} \mathrm{mL}^{-1}$ crosslinking solution.

MS Preparation and Surface Modification. MS particles were synthesized according to a modified literature method. ${ }^{23}$ To aid polymer loading, MS particles were functionalized with a layer of primary amine by APTES modification to promote polymer adhesion and infiltration. In this process, the MS particles were dispersed in ethanol with a concentration of $30 \mathrm{mg} \mathrm{mL}^{-1}$ by sonication for $10 \mathrm{~min}$, before ammonia and APTES were added to the suspension. The volume ratio of ethanol:ammonia:APTES was fixed at 20:1:1 and the suspension was allowed to stir overnight. APTES-modified MS particles were finally washed with ethanol and Milli-Q water.

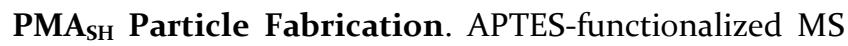
(2.5 mg) was added to a PMA-PDA ( $\left.5 \mathrm{mg} \mathrm{mL}^{-1}\right)$ in $\mathrm{NaOAc}$ (o.1 M, pH 5.0) solution at a mass ratio of 0.3:1 polymer:MS. This solution was vortexed and sonicated thoroughly, and allowed to incubate overnight with constant agitation. Infiltrated MS was then washed via centrifugation three times ( $3 \mathrm{~min}, 1100 \mathrm{~g}$ ) and resuspended in acetate buffer. The pellet was divided evenly $(5 \times 0.5 \mathrm{mg} \mathrm{MS})$, $200 \mu \mathrm{L}$ of $\mathrm{PMA}_{\mathrm{SH}}$ solution added at different concentrations in PBS buffer (2.0, 1.0, 0.5, 0.2, $\left.0.1 \mathrm{mg} \mathrm{mL}^{-1}\right)$, and incubated overnight. After two washing cycles with Milli-Q water, the MS templates were dissolved with $50 \mu \mathrm{L}$ of a 5 M HF solution (pH 1.0). Caution! HF is highly toxic. Extreme care should be taken when handling HF solution and only small quantities should be prepared. The resultant $\mathrm{PMA}_{\mathrm{SH}}$ particles were centrifuged $(3 \mathrm{~min}$, $3000 \mathrm{~g}$ ) and washed three times with PBS buffer. A schematic representation of this process is illustrated in Figure $1 \mathrm{~A}$.

Characterization Methods. Hydrogel microparticles were imaged on an Olympus IX $_{71}$ inverted fluorescence microscope (100 $\times / 1.40$ oil objective, Olympus). Confocal laser scanning microscopy (CLSM) experiments were performed on a Leica TCS $\mathrm{SP}_{2}$ confocal system equipped with an HCX PL APO lbd.BL $63 \times 1.4$ NA oil objective. For electrostatic immobilization, microscope coverglasses (ProSciTech) were immersed into PEI solutions ( $1 \mathrm{~g} \mathrm{~L}^{-1}, 0.5$ $\mathrm{M} \mathrm{NaCl}$ ) for $15 \mathrm{~min}$, rinsed with water, and dried under a nitrogen stream. Transmission electron microscopy experiments (TEM, Philips CM12o BioTWIN, operated at 120 $\mathrm{kV})$ were performed by placing samples in buffer $(2 \mu \mathrm{L})$ onto Formvar-coated copper grids and allowed to air-dry. Milli-Q water was dropped on the copper grid to dissolve precipitated buffer salts and tissue paper was used to remove the droplet. Non-contact mode atomic force microscopy imaging was performed in air using a JPK Nanowizard II (JPK Instruments AG, Berlin, Germany) with soft tapping-mode cantilevers $\left(5 \mathrm{~N} \mathrm{~m}^{-1}\right.$, Tap150-G, Budget Sensors, Bulgaria). Images were post-treated using JPK image processing software and algorithms. A three-point $1^{\text {st }}$ degree polynomial fit was first subtracted from the image, and then a polynomial fit subtracted from each scan line independently, excluding capsule regions. Prior to measurement, cantilevers and glass substrates were immersed in a 30 vol\% isopropanol solution, followed by Milli-Q water, and further cleaned using oxygen plasma for $180 \mathrm{~s}$ (Harrick Plasma, o.1 L min ${ }^{-1}, 29.6 \mathrm{~W}$, $300 \mathrm{mTorr}$ ). Cleaned substrates were also treated with a monolayer of PEI to promote particle immobilization. To determine the microparticle concentration of the particle dispersions, flow cytometry analysis was performed on a CyFlow Space (Partec GmbH) flow cytometer using an excitation wavelength of $488 \mathrm{~nm}$. For each measurement at least 15000 events were analyzed. The fluorescence emission intensity of $\mathrm{PMA}_{\mathrm{SH}}$ solutions was measured using a Horiba Fluorolog-3 Model FL3-22 spectrofluorometer (Jobin Yvon Inc., USA) equipped with a HgXe lamp (excitation wavelength, $488 \mathrm{~nm}$; emission wavelength, $519 \mathrm{~nm}$; increment size, $1 \mathrm{~nm}$; excitation and emission slit widths, $1 \mathrm{~nm}$ ).

AFM Force Spectroscopy. Mechanical characterization of the PMA $\mathrm{A}_{\mathrm{SH}}$ particles was performed using a JPK Nanowizard II AFM. The measurement temperature was held at $23 \pm 1{ }^{\circ} \mathrm{C}$ using a thermo-regulated BioCell add-on (JPK Instruments $A G)$. High-reflectance cantilevers were used with nominal spring constants ranging from 0.03 to 0.09 $\mathrm{N} \mathrm{m}^{-1}$ (CSC38/Cr-Au, MikroMasch, Estonia). Substrates and cantilevers were cleaned and prepared as described above. Cantilevers were calibrated in air on the PEIcoated substrates prior to measurement, using the thermal noise method. ${ }^{24}$ After calibration, $1 \mu \mathrm{L}$ of a diluted (10×, PBS) particle suspension was dispensed onto the substrate and ca. $150 \mu \mathrm{L}$ of PBS buffer was added. The cantilever was then fully immersed in the droplet, and the inverse optical lever sensitivity (InvOLS) of the cantilever remeasured in buffer. Particles immobilized on the surface could be visualized in situ using a Leica DMI $4000 \mathrm{~B}$ optical microscope and $40 \times$ objective (Leica Microsystems GmbH, Wetzlar, Germany). Force spectroscopy measurements were then made on the hydrogel particles by optically aligning the cantilever tip above individual particles, and initiating an approach-retract force cycle with a constant piezo velocity of $3.0 \mu \mathrm{m} \mathrm{s}^{-1}$. It was seen that when deforming immobilized particles, the forcedisplacement gradient differed significantly from the InvOLS of the PEI-coated substrate. For force mapping analysis, a piezo retract time of $0.3 \mathrm{~s}$ was also used between individual measurements. A schematic representation of this process is shown in Figure $1 B$.

\section{RESULTS AND DISCUSSION}

Fabrication and Characterization of Nanoporous PMA $_{\text {SH }}$ Particles. To construct polymer particles with a high loading capacity, MS particles with a bimodal pore structure (smaller mesopores in the $2-3 \mathrm{~nm}$ range and larger mesopores between 10-40 $\mathrm{nm}$ ) were used as templates. Nitrogen adsorption data previously reported indicated that the MS particles had a surface area of $893 \mathrm{~m}^{2} \mathrm{~g}^{-1}$ 
and a pore volume of $1.34 \mathrm{~cm}^{3} \mathrm{~g}^{-1} .5$ Previous transmission electron microscopy (TEM) and scanning electron microscopy (SEM) studies revealed that the mean diameter of the MS particles was ca. $800 \mathrm{~nm} .^{5}$ These MS particles with large mesopores and extensive surface area have proven to be excellent templates for the assembly of polymer particles from large molecular weight macromolecules, for biological applications., 5,22
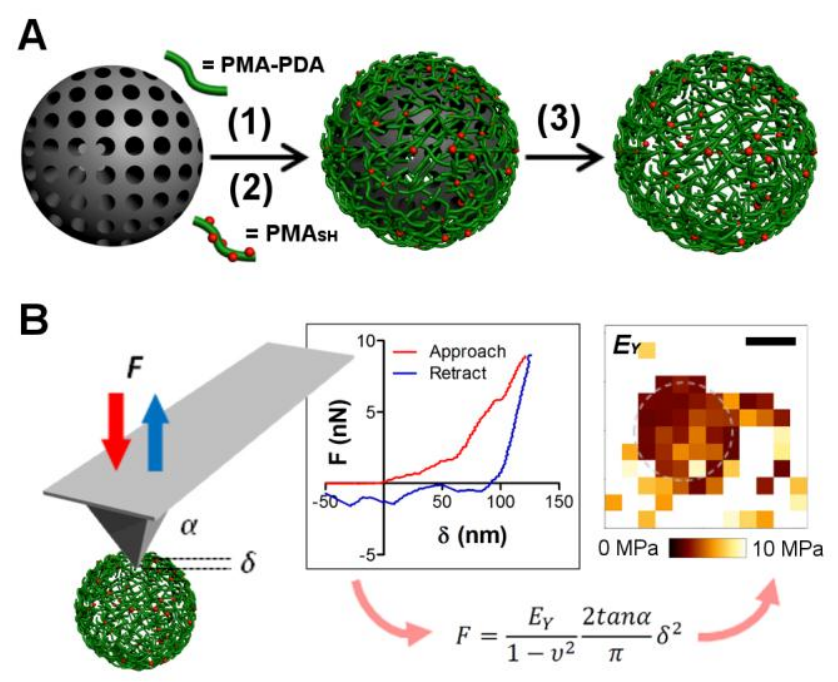

Figure 1. (A) $P M A_{S H}$ particle fabrication and (B) single particle AFM force measurement. PMA-PDA is added to MS (1), then fluorescently labeled $\mathrm{PMA}_{S H}$ is introduced and oxidized to form disulfide cross-links (2). The MS is removed via HF treatment to yield stable PMA $\mathrm{SH}_{\mathrm{SH}}$ replica particles (3). In B a representative force-deformation (F- $\delta$ ) curve is shown, along with the Sneddon equation and example Young's modulus map for a single hydrogel particle (scale bar $500 \mathrm{~nm}$ ).

From integration of the corresponding ${ }^{1} \mathrm{H}-\mathrm{NMR}$ spectrum (SI, Figure $\mathrm{S}_{1}$ ), it was determined that PMA-PDA contained $14 \mathrm{~mol} \%$ thiol functionality. Loading of the modified MS with PMA-PDA, crosslinking with variable concentrations of PMA $\mathrm{PMH}_{\mathrm{SH}}$ as per Scheme $\mathrm{S}_{1}(\mathrm{SI})$, and dissolution of the MS core, led to the formation of stable replica particles (Figure 1). As can be seen from fluorescence microscopy (Figure 2) and CLSM (SI, Figure S2) images, there was successful loading of the fluorescently labeled $\mathrm{PMA}_{\mathrm{SH}}$ into the MS template particles, and subsequent covalent attachment. Due to the absence of hydrogen bonding and additional charge repulsion above 


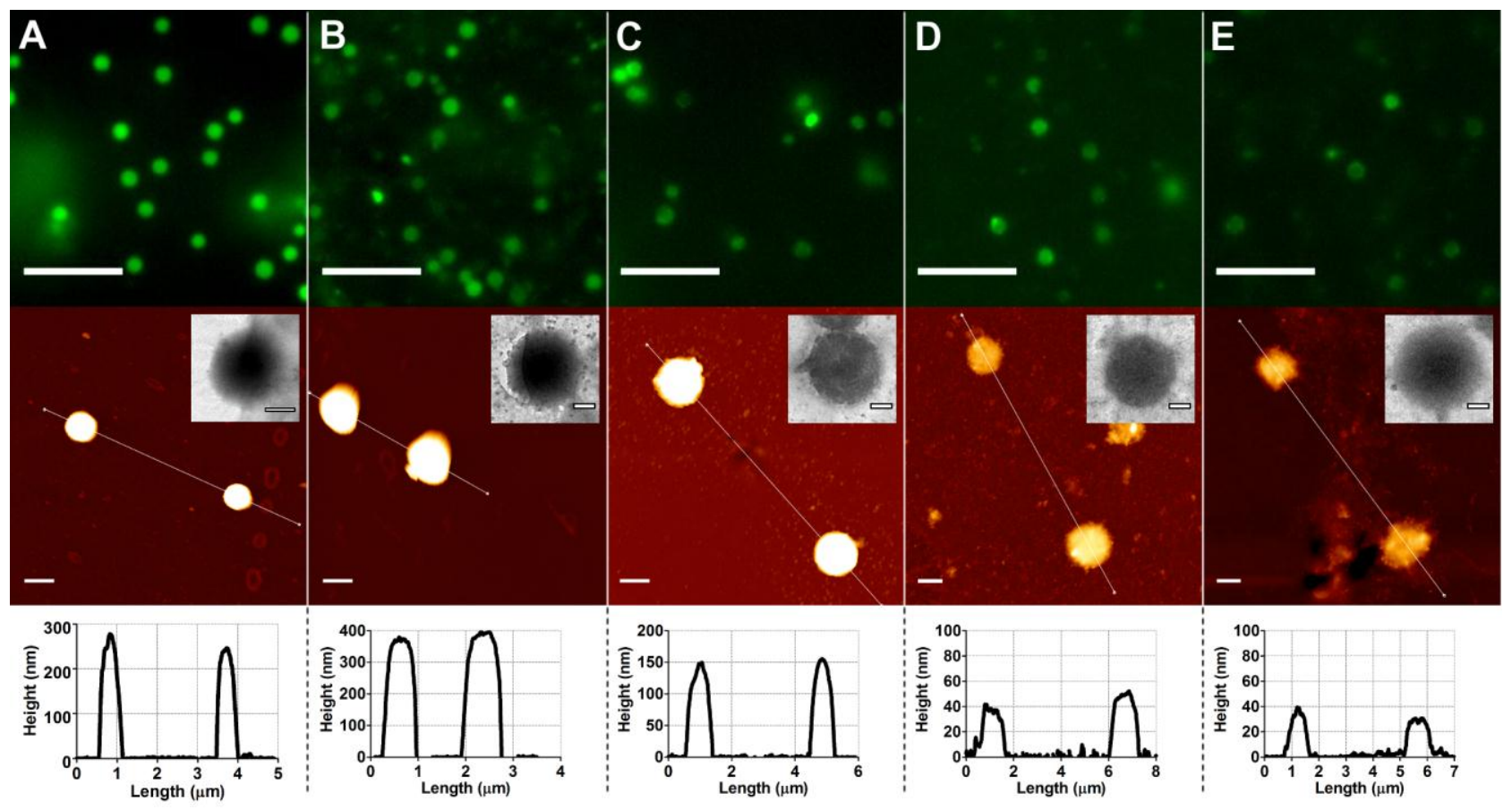

Figure 2. Characterization of nanoporous $\mathrm{PMA}_{\mathrm{SH}}$ particles with varying cross-linker concentration: (A) 2.0, (B) 1.0, (C) o.5, (D) 0.2 , and (E) $0.1 \mathrm{mg} \mathrm{mL}^{-1}$. Fluorescence microscopy (top, scale bar $\left.10 \mu \mathrm{m}\right)$, AFM height (middle, scale bar $1 \mu \mathrm{m}$ ), and TEM images (middle inset, scale bar $500 \mathrm{~nm}$ ) are shown. AFM height profiles (bottom) are shown for corresponding AFM height images.

the dissociation constant of PMA $\left(\mathrm{p} K_{\mathrm{a}} \sim 5.65\right),{ }^{25}$ particles without an established covalent crosslinking network would disintegrate at $\mathrm{pH}$ 7.4. The fluorescence intensity of individual particles was observed to decrease with decreasing $\mathrm{PMA}_{\mathrm{SH}}$ concentration from CLSM intensity profiles (SI, Figure S2), while a slight decrease in particle concentration was also observed by fluorescence microscopy (Figure 2). This is likely due to a decrease in particle density with decreasing cross-linker concentration, leading to removal of a small fraction of particles during washing steps. This reasoning can be correlated to TEM and AFM imaging results (Figure 2). TEM images show that particles fabricated with a greater concentration of crosslinking PMA $_{S H}$ exhibit higher contrast, while AFM height profiles show a general trend of increasing airdried particle height-to-diameter ratio with increasing $\mathrm{PMA}_{\mathrm{SH}}$ concentration. It can be reasoned that this is due to a greater mass of material in particulate systems fabricated with greater concentrations of $\mathrm{PMA}_{\mathrm{SH}}$ cross-linker. For 0.1 and $0.2 \mathrm{mg} \mathrm{mL}^{-1} \mathrm{PMA}_{\mathrm{SH}}$ hydrogel particles, a greater concentration of 'capsule-like' particles could be observed via fluorescence microscopy, where the fluorescence intensity was greater around the circumference of the particle due to a dominating surface localization of fluorescently labeled $\mathrm{PMA}_{\mathrm{SH}}$. While small height variations were observed between 2.0 and $1.0 \mathrm{mg} \mathrm{mL}^{-1} \mathrm{PMA}_{\mathrm{SH}}$, there were considerable optical and height differences for dehydrated particles ranging between 0.1 to $1.0 \mathrm{mg} \mathrm{mL}^{-1}$ of $\mathrm{PMA}_{\mathrm{SH}}$ cross-linker.

AFM Force Spectroscopy Measurements. Highresolution force maps were performed on single $\mathrm{PMA} \mathrm{A}_{\mathrm{SH}}$ particles immobilized onto the PEI-coated substrate, as illustrated in Figure $1 \mathrm{~B}$. Approximately $60 \times 60$ force spectra were collected over a $1.5 \times 1.5 \mu \mathrm{m}$ area, and the effect on nanoscale force response was studied. Indentation was performed using a conical tipped cantilever, which was previously calibrated to elucidate the spring constant. Raw AFM voltage-displacement data was processed using JPK data processing software to subtract the zero-force baseline, shift the data along the deformation axis to zero the initial contact point, and to extract data points. The effect of cantilever bending during sample compression was removed to give true sample force-deformation $(F / \delta)$ data, as outlined below:

$$
\delta=\text { Displacement }-\left(F / k_{c}\right)
$$

where $k_{c}$ is the spring constant of the cantilever. For an incompressible material, the force increases with no observable change in deformation, while for softer samples the gradient is reduced due to the compliance of the material. Due to adhesive contact between probe and sample, and possible viscoelastic effects and associated hysteresis, only the approach force curve was analyzed (Figure 3). ${ }^{26}$

The Young's modulus $\left(E_{Y}\right)$ was determined over the first $10 \%$ of total deformation using the Hertz-Sneddon relationship for a conical indenter:

$$
F=\frac{E_{Y}}{1-v^{2}} \frac{2 \tan \alpha}{\pi} \delta^{2}
$$

where $v$ is the Poisson ratio and $\alpha$ the semi-opening angle of the conical indenter. ${ }^{27}$ The Poisson ratio was assumed 
to be 0.5 , a reasonable value for incompressible isotropic elastic materials, while $\alpha$ was determined as $15.2 \pm 0.4^{\circ}$ through scanning electron microscopy analysis of the cantilever batch (data not shown). It was important to only analyze data in the small deformation regime to negate the effect of imparting excessive axial strain on the system during measurement.

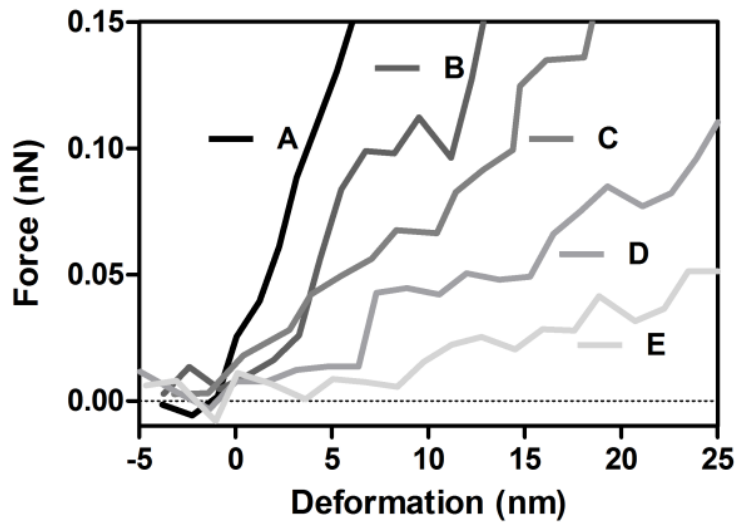

Figure 3. Representative $F / \delta$ curves for hydrogel particles cross-linked with (A) 2.0, (B) 1.0, (C) 0.5, (D) 0.2, and (E) 0.1 $\mathrm{mg} \mathrm{mL}^{-1} \mathrm{PMA}_{\mathrm{SH}}$ cross-linker.

$E_{Y}$ values determined using JPK data processing software were arranged into a matrix and then graphically represented in three-dimensions (Figure 4). For clarity, large $E_{Y}$ values corresponding to substrate incompressibility were removed. It could be seen that the overall particle response was vastly altered depending on the concentration of added cross-linker. The most stable particles were obtained for 1.0 and 2.0 $\mathrm{mg} \mathrm{mL}^{-1} \mathrm{PMA}_{\mathrm{SH}}$ cross-linker, as seen in Figures $4 \mathrm{~A}$ and $\mathrm{B}$, where both force indentation and electrostatic interaction with PEI did not lead to a significant deformation or spreading of the particles. This led to a homogeneous $E_{Y}$ distribution over the particle surface. Decreasing the initial PMA $\mathrm{ASH}_{\mathrm{SH}}$ concentration to 0.5 $\mathrm{mg} \mathrm{mL}^{-1}$ led to the formation of less stable particles, with pronounced polymer regions in the middle of the particle (Figure ${ }_{4} C$ ) corresponding to maximum $E_{Y}$ zones. Particle systems with 0.1 and $0.2 \mathrm{mg} \mathrm{mL}^{-1} \mathrm{PMA}_{\mathrm{SH}}$ cross-linker (Figures $4 \mathrm{D} / \mathrm{E}$ ), however, are significantly deformed upon interaction with the PEI-coated substrate, as was seen for the AFM height images in Figure 2. In addition, it was observed that with increasing cross-linker concentration, Young's modulus zones became more structured within the center of the particle. Piezo height maps (Figure 4, inset) were consistent at ca. $600 \mathrm{~nm}$ for 2.0, 1.0 and 0.5 $\mathrm{mg} \mathrm{mL} \mathrm{m}^{-1}$ cross-linker particles; however, the height profiles decreased substantially for 0.2 and $0.1 \mathrm{mg} \mathrm{mL}^{-1}$ particles (ca. $300 \mathrm{~nm}$ and $80 \mathrm{~nm}$, respectively) due to network and particle instability at these concentrations. When compared to dry particle AFM height images in Figure 2, we can see that the nanoporous particles swell considerably when hydrated, consistent with observations previously reported. ${ }^{15}$

The sensitivity of single hydrogel particles to indentation frequency and velocity was studied to investigate plastic deformation and viscoelastic behavior, respectively (SI, Figure $\mathrm{S}_{3} \mathrm{~A}$ and $\mathrm{B}$ ). The elastic modulus was independent of the indentation frequency for stiffer systems, softer particles, however, showed an increase of the modulus after ca. 10 indentations, presumably due to the increase in material compliance leading to irreversible damage of the particle thereby increasing substrate/probe interaction. The indentation velocity was observed to have negligible effect in $E_{Y}$ for particle systems until a piezo velocity of $3.0 \mu \mathrm{m} \mathrm{s}^{-1}$, where a significant inconsistency was observed in the modulus at velocities exceeding this. For the $1.0 \mathrm{mg} \mathrm{mL}^{-1}$ system, the influence of increasing the temperature from 23 to $37^{\circ} \mathrm{C}$ on the particle $E_{Y}$ was also investigated (SI, Figures $\mathrm{S}_{3} \mathrm{C}$ and D). A controlled increase of the buffer temperature led to a considerable decrease in the average surface $E_{Y}$ of ca. 30\%. This is possibly a result of increased chain mobility in these systems at higher temperatures, in combination with a mobile disulfide crosslinking network. This sensitivity to temperature is an important consideration when considering these soft hydrogel materials for biomedical applications.

A

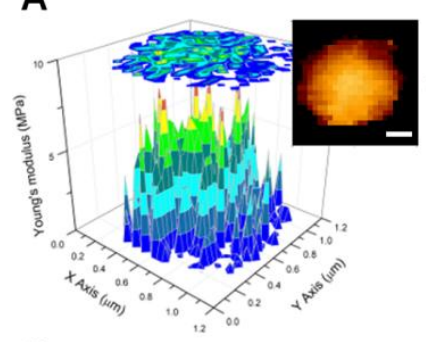

C
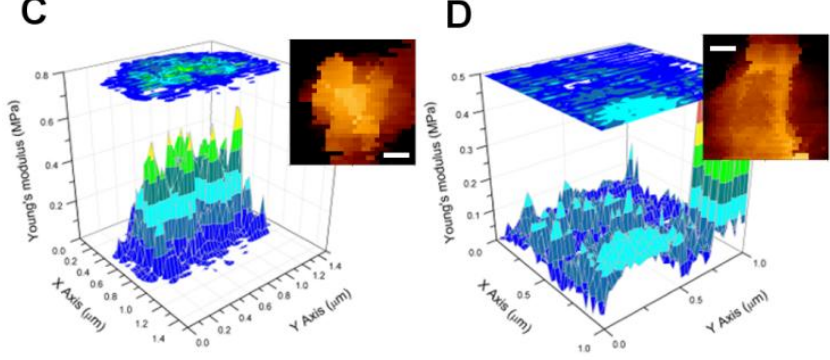

$\mathbf{E}$

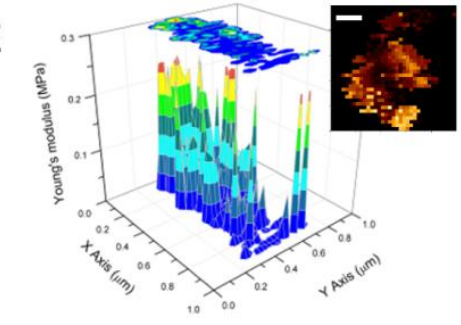

Figure 4. High-resolution force tomography maps and contour plots for (A) 2.0, (B) 1.0, (C) 0.5, (D) 0.2, and (E) $0.1 \mathrm{mg}$ $\mathrm{mL}^{-1}$ cross-linker. Insets show piezo height maps for the particles during the force scan (scale bars are $200 \mathrm{~nm}$ ). 
Single particles in each system were then investigated to collect statistically relevant mechanical information on the particle suspensions. To achieve representative $E_{Y}$ values for the hydrogel particles, at least 10 different particles were analyzed for each sample, allowing for standard errors in the data set to be determined. The particle stiffness was obtained through linear-regression analysis of the small-deformation regime of the obtained force curves. Additionally, the Young's modulus was calculated as previously described (Equation 2), with results shown in Figure 5. The magnitude of these results correspond well with literature values; the $E_{Y}$ for substrateimmobilized hydrogel microparticles was found to range between $80 \mathrm{kPa}$ to $2.5 \mathrm{MPa}$ using a similar methodology. ${ }^{15}$ It was also observed that the mechanical properties $\left(k, E_{Y}\right)$ gradually stabilize with increasing cross-linker concentration.

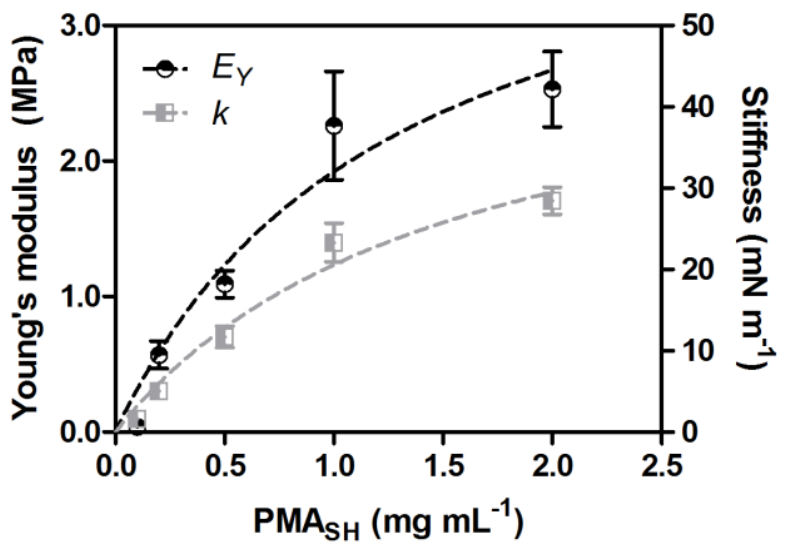

Figure 5. Mean mechanical parameters ( $E_{Y}$ and $k$ ) derived from $F / \delta$ analysis for the hydrogel microparticles. Error bars represent the standard error, while dotted lines show nonlinear regression fits of Equation 3.

The results presented in Figure 5 are consistent with our previous findings ${ }^{19}$ and trends reported in literature: we found a similar trend for nanoporous poly(L-glutamic acid) particles for vaccine delivery, 5 while Senger and coworkers observed a plateau relationship between $E_{Y}$ and EDC cross-linker concentration for planar polyelectrolyte films measured using colloidal probe AFM.18 The mechanical results shown in Figure 5 for $k$ and $E_{Y}$ were modeled using the Langmuir isotherm (Equation 3):

$$
\Gamma=\Gamma_{\max } \frac{K c}{1+K c}
$$

where $\Gamma$ is the amount adsorbed, $\Gamma_{\max }$ is the maximum amount adsorbed, $c$ is the concentration, and $K$ is the Langmuir equilibrium constant. ${ }^{28}$ The Langmuir isotherm, in effect, relates molecular adsorption to concentration. We propose that this relationship is analogous to the polymer loading mechanism into the MS templates investigated here. By increasing the concentration of PMA $_{S H}$ above the PMA-PDA loaded-MS surface, the amount of $\mathrm{PMA}_{\mathrm{SH}}$ adsorbed is controlled according to Equation 3. Evidence of this is seen for 0.1 and $0.2 \mathrm{mg} \mathrm{mL}^{-1}$ $\mathrm{PMA}_{\mathrm{SH}}$ particles, where the lower adsorption driving force of $\mathrm{PMA}_{\mathrm{SH}}$ results in the formation of an increasing number of 'capsule-like' particles (Figure 2). It may therefore be hypothesized that the amount of adsorbed cross-linker directly mediates the mechanical properties of the hydrogel particle systems.

Quantification of Cross-linker Amount. To prove the direct relationship between adsorbed mass of PMA $\mathrm{P}_{\mathrm{SH}}$ and the mechanical properties of the particle systems, we sought to quantify the average mass of cross-linker within a single hydrogel particle. The concentration of particles in each suspension was first quantified using flow cytometry (SI, Figure $\mathrm{S}_{4}$ ). We observed that the mean FLi fluorescence signal decreased with $\mathrm{PMA}_{\mathrm{SH}}$ cross-linker concentration. This is expected when viewed in conjunction with our previous results, where we also observe a decrease in particle density and fluorescence intensity with crosslinking (Figure 2). It was observed that the particle concentration tended to decrease with cross-linker concentration; however, the concentration was more variable for the softer 0.1-0.5 $\mathrm{mg} \mathrm{mL}^{-1} \mathrm{PMA}_{\mathrm{SH}}$ cross-linker particles. It was reasoned that this was due to difficulties in supernatant removal for low-density hydrogel particles during washing steps. Irrespective of this, the loss of particles was found to have negligible effect on the average mass for $\mathrm{PMA}_{\mathrm{SH}}$ covalently bound in the hydrogel particles.

To examine the solutions using fluorescence spectrofluorometry, $\mathrm{PMA}_{\mathrm{SH}}$ hydrogel particles were disassembled into their constituent polymers using a strong reducing agent (DTT). DTT is particularly effective at cleaving disulfide bonds at $\mathrm{pH}$ values greater than 7.o. A $10 \mathrm{M}$ DTT (10 $\mu \mathrm{L}, \mathrm{MOPS}$ ) solution and $150 \mu \mathrm{L}$ of buffer was added to the particle dispersion $(40 \mu \mathrm{L})$ to give a final concentration of $0.5 \mathrm{M}$ DTT. The $\mathrm{pH}$ was then adjusted to 8.o using 1.o $\mathrm{M} \mathrm{NaOH}$. Gentle sonication aided in rapid cleavage of the covalent cross-links, and complete particle disassembly was observed using a combination of fluorescence microscopy and flow cytometry (SI, Figure $\mathrm{S}_{5}$ ). 
A

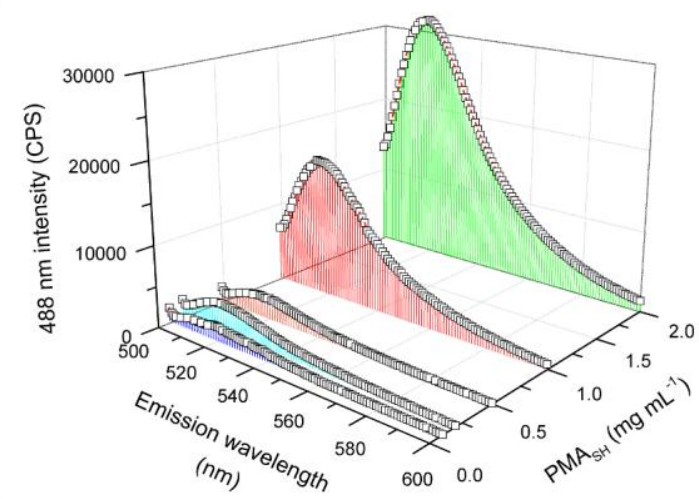

B

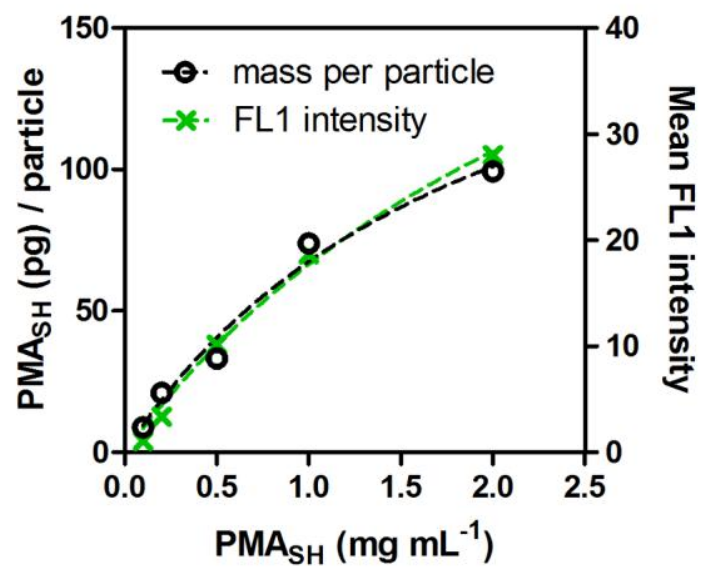

Figure 6. (A) Fluorescence emission spectra immediately after $\mathrm{PMA}_{\mathrm{SH}}$ particle degradation at an excitation wavelength of $488 \mathrm{~nm}$. (B) Mass of cross-linker per nanoporous particle to added cross-linker concentration. Also shown is the mean FL1 fluorescence intensity determined using flow cytometry. Dotted lines for each data set show non-linear regression fits of Equation 3.

Fluorescence spectra were immediately measured for each particle system (Figure 6A), and the intensity converted into $\mathrm{PMA}_{\mathrm{SH}}$ amount using cytometric population data and a fluorescence calibration curve for fluorescently labeled $\mathrm{PMA}_{\mathrm{SH}}$ (SI, Figure S6). The results for PMA $\mathrm{PH}_{\mathrm{SH}}$ mass per particle (Figure 6B) show a similar trend to that of mean FL1 intensity observed in cytometry results, and also to the stabilizing behavior of the Langmuir isotherm (Figure 5). The experiment was repeated with a separate particle batch, and while particle concentrations were variable, the trends and values for the mass of cross-linker per particle were found to be equivalent.

The mass of cross-linker per particle in each crosslinking system was then correlated to the mechanical properties of the particles (Figure 7). It was found that there was a linear dependency for both the stiffness and Young's modulus of the particles. This is analogous to the result reported by Picart and co-workers for polyelectrolyte multilayer films cross-linked using EDC; a linear relationship was observed between $E_{Y}$ and covalent cross-link density. ${ }^{20}$ It can therefore be reasoned that the mechanical properties of the nanoporous hydrogel particles are directly related to the network density of the material structure, and that the mechanical limitation for these systems is solely based on the amount of polymer material that can be adsorbed in the porous support structure. The mechanisms of porous replica particle formation discussed here may assist in the design of 'tailor-made' hydrogel particles for biomedical as well as other applications.

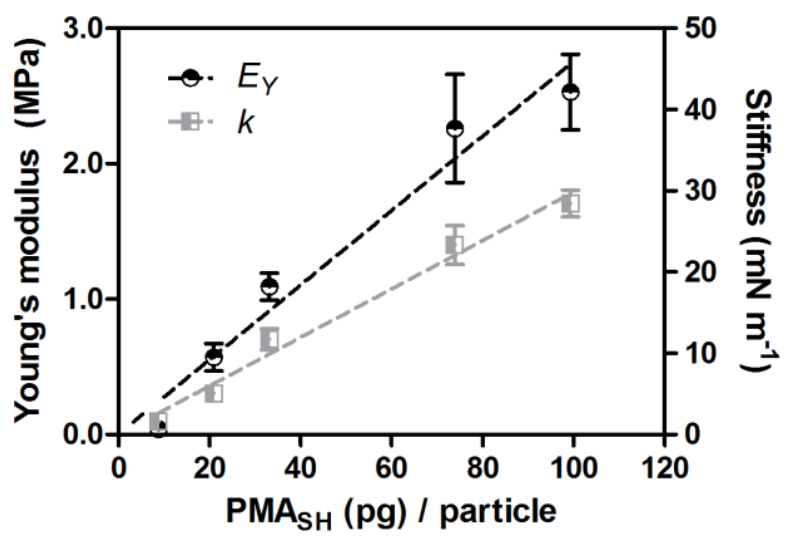

Figure 7. Mechanical properties ( $E_{Y}$ and $k$ ) of nanoporous $\mathrm{PMA}_{\mathrm{SH}}$ particles as a function of cross-linker mass. Dotted lines represent linear regression fits to the data, while error bars represent the standard error.

\section{CONCLUSIONS}

Nanoporous hydrogel $\mathrm{PMA}_{\mathrm{SH}}$ particles were fabricated using a mesoporous silica templating method. The hydrogel particles were stabilized using varying amounts of cross-linker and were characterized using optical and force microscopy techniques. It was found that the mechanical properties and structural integrity could be effectively tuned through variation of the cross-linker concentration. High-resolution force mapping confirmed particle integrity results through investigation of the nanoscale force response of individual particles. In addition, fluorescent tagging of the cross-linker in combination with flow cytometry and fluorescence spectroscopy allowed for accurate quantification of an average cross-linker mass per particle.

We observed a linear dependence between the mechanical properties of hydrogel particles and the determined amount of adsorbed cross-linker material per particle. The mechanical properties of nanoporous polymer replica particles follow a Langmuir isotherm, and this can be used to predict particle properties. This is advantageous when compared to previous efforts, due to the ability to accurately quantify the amount of cross-linker in the system. This work will enable researchers to reason similar mechanical behavior in adsorption based polymer sys- 
tems, and aid in the design of next-generation soft microparticles for biomedical applications.

\section{ASSOCIATED CONTENT}

\section{Supporting Information.}

PMA-PDA ${ }^{1} \mathrm{H}-\mathrm{NMR}$ spectrum and characterization, polymer crosslinking scheme, CLSM images and intensity profiles, frequency and piezo velocity mechanical tests, temperature dependency test, flow cytometer scatter plots, particle counting and degradation results, and calibration curve for fluorescently labeled $\mathrm{PMA}_{\mathrm{SH}}$. This material is available free of charge via the Internet at http://pubs.acs.org

\section{AUTHOR INFORMATION}

\section{Corresponding Author}

* E-mail: fcaruso@unimelb.edu.au, Tel +61-3-8344-3461, Fax $+61-3-8344-4153$.

\section{Author Contributions}

The manuscript was written through contributions of all authors. All authors have given approval to the final version of the manuscript.

\section{Notes}

The authors declare no competing financial interest.

\section{ACKNOWLEDGMENT}

This work was supported by the Australian Research Council under the Australian Laureate Fellowship (F.C., 120100030) and Discovery Project schemes (F.C., 130101846). J.C. is the recipient of an Australian Research Council Super Science Fellowship (FS110200025). J.C. also acknowledges the support of the Early Career Research Grant from the University of Melbourne.

\section{ABBREVIATIONS}

AF488, cadaverine functionalized Alexa Fluor 488; AFM, atomic force microscopy; APTES, (3-aminopropyl) triethoxysilane; CLSM, confocal laser scanning microscopy; DTT, dithiothreitol; EDC, 1-ethyl-3-(3-dimethylaminopropyl) carbodiimide; HF, hydrofluoric acid; MES, 2-(N-morpholino) ethanesulfonic acid; MOPS, 3-( $N$-morpholino) propanesulfonic acid; MS, mesoporous silica; MWCO, molecular weight cutoff; NaOAc, sodium acetate; NMR, nuclear magnetic resonance; PBS, phosphate buffered saline; PMA, poly(methacrylic acid); SEC, size exclusion chromatography; SEM, scanning electron microscopy; TEM, transmission electron microscopy.

\section{REFERENCES}

(1) LaVan, D. A.; McGuire, T.; Langer, R. Small-Scale Systems for In Vivo Drug Delivery. Nat. Biotechnol. 2003, 21, 1184-1191.

(2) Farokhzad, O. C.; Langer, R. Impact of Nanotechnology on Drug Delivery. ACS Nano 2009, 3, 16-20.

(3) Wang, A. Z.; Langer, R.; Farokhzad, O. C. Nanoparticle Delivery of Cancer Drugs. Annu. Rev. Med. 2012, 63, 185-198.

(4) Petros, R. A.; DeSimone, J. M. Strategies in the Design of Nanoparticles for Therapeutic Applications. Nat. Rev. Drug Disc. 2010, 9, 615-627.
(5) Cui, J.; De Rose, R.; Best, J. P.; Johnston, A. P.; Alcantara, S.; Liang, K.; Such, G. K.; Kent, S. J.; Caruso, F. Mechanically Tunable, Self-Adjuvanting Nanoengineered Polypeptide Particles. Adv. Mater. 2013, DOI:10.1002/adma.201300981.

(6) Andreasen, S.; Chong, S.-F.; Wohl, B. M.; Goldie, K. N.; Zelikin, A. N. Poly(vinyl alcohol) Physical Hydrogel Nanoparticles, Not Polymer Solutions, Exert Inhibition of Nitric Oxide Synthesis in Cultured Macrophages. Biomacromolecules 2013 , DOI:10.1021/bm400369u.

(7) Yan, Y.; Johnston, A. P.; Dodds, S. J.; Kamphuis, M. M.; Ferguson, C.; Parton, R. G.; Nice, E. C.; Heath, J. K.; Caruso, F. Uptake and Intracellular Fate of Disulfide-Bonded Polymer Hydrogel Capsules for Doxorubicin Delivery to Colorectal Cancer Cells. ACS Nano 2010, 4, 2928-2936.

(8) Gupta, B.; Levchenko, T. S.; Torchilin, V. P. Intracellular Delivery of Large Molecules and Small Particles by Cell-Penetrating Proteins and Peptides. Adv. Drug Deliv. Rev. 2005, 57, 637-651.

(9) Becker, A. L.; Johnston, A. P.; Caruso, F. Layer-By-LayerAssembled Capsules and Films for Therapeutic Delivery. Small 2010, 6, 1836-1852.

(10) Best, J. P.; Yan, Y.; Caruso, F. The Role of Particle Geometry and Mechanics in the Biological Domain. Adv. Healthcare Mater. 2012, $1,35-47$.

(11) Devarajan, P. V.; Jindal, A. B.; Patil, R. R.; Mulla, F.; Gaikwad, R. V.; Samad, A. Particle Shape: A New Design Parameter for Passive Targeting in Splenotropic Drug Delivery. J. Pharm. Sci. 2010, 99, 2576-2581.

(12) Geng, Y.; Dalhaimer, P.; Cai, S.; Tsai, R.; Tewari, M.; Minko, T.; Discher, D. E. Shape Effects of Filaments Versus Spherical Particles in Flow and Drug Delivery. Nat. Nanotechnol. 2007, 2, 249255.

(13) Beningo, K. A.; Wang, Y.-1. Fc-Receptor-Mediated Phagocytosis is Regulated by Mechanical Properties of the Target. J. Cell Sci. 2002, $115,849-856$

(14) Banquy, X.; Suarez, F.; Argaw, A.; Rabanel, J.-M.; Grutter, P.; Bouchard, J.-F.; Hildgen, P.; Giasson, S. Effect of Mechanical Properties of Hydrogel Nanoparticles on Macrophage Cell Uptake. Soft Matter 2009, 5, 3984-3991.

(15) Burmistrova, A.; Richter, M.; Uzum, C.; von Klitzing, R. Effect of Cross-Linker Density of P(NIPAM-co-AAc) Microgels at Solid Surfaces on the Swelling/Shrinking Behaviour and the Young's Modulus. Colloid Polym. Sci. 2011, 289, 613-624.

(16) Elsner, N.; Kozlovskaya, V.; Sukhishvili, S. A.; Fery, A. pHTriggered Softening of Crosslinked Hydrogen-Bonded Capsules. Soft Matter 2006, 2, 966-972.

(17) Schneider, A.; Francius, G.; Obeid, R.; Schwinté, P.; Hemmerlé, J.; Frisch, B.; Schaaf, P.; Voegel, J.-C.; Senger, B.; Picart, C. Polyelectrolyte Multilayers with a Tunable Young's Modulus: Influence of Film Stiffness on Cell Adhesion. Langmuir 2006, 22, $1193-1200$

(18) Francius, G.; Hemmerlé, J.; Ohayon, J.; Schaaf, P.; Voegel, J. C.; Picart, C.; Senger, B. Effect of Crosslinking on the Elasticity of Polyelectrolyte Multilayer Films Measured by Colloidal Probe AFM. Microsc. Res. Techniq. 2006, 69, 84-92.

(19) Liang, K.; Such, G. K.; Zhu, Z.; Dodds, S. J.; Johnston, A. P. R.; Cui, J.; Ejima, H.; Caruso, F. Engineering Cellular Degradation of Multilayered Capsules through Controlled Cross-Linking. ACS Nano 2012, 6, 10186-10194.

(20) Boudou, T.; Crouzier, T.; Auzély-Velty, R.; Glinel, K.; Picart, C. Internal Composition Versus the Mechanical Properties of Polyelectrolyte Multilayer Films: The Influence of Chemical CrossLinking. Langmuir 2009, 25, 13809-13819.

(21) Wang, Y.; Price, A. D.; Caruso, F. Nanoporous Colloids: Building Blocks for a New Generation of Structured Materials. $J$. Mater. Chem. 2009, 19, 6451-6464.

(22) Tan, J.; Wang, Y.; Yip, X.; Glynn, F.; Shepherd, R. K.; Caruso, F. Nanoporous Peptide Particles for Encapsulating and Releasing 
Neurotrophic Factors in an Animal Model of Neurodegeneration. $A d v$. Mater. 2012, 24, 3362-6.

(23) Wang, J.-G.; Zhou, H.-J.; Sun, P.-C.; Ding, D.-T.; Chen, T.-H. Hollow Carved Single-Crystal Mesoporous Silica Templated by Mesomorphous Polyelectrolyte-Surfactant Complexes. Chem. Mater. 2010, 22, 3829-3831.

(24) Attard, P. Measurement and Interpretation of Elastic and Viscoelastic Properties with the Atomic Force Microscope. J. Phys.: Condens. Matter 2007, 19, 473201.

(25) Porasso, R. D.; Benegas, J. C.; van den Hoop, M. A. Chemical and Electrostatic Association of Various Metal Ions by Poly(acrylic acid) and Poly(methacrylic acid) as Studied by Potentiometry. $J$. Phys. Chem. B 1999, 103, 2361-2365.

(26) Wiedemair, J.; Serpe, M. J.; Kim, J.; Masson, J.-F.; Lyon, L. A.; Mizaikoff, B.; Kranz, C. In-situ AFM Studies of the Phase-Transition Behavior of Single Thermoresponsive Hydrogel Particles. Langmuir 2007, 23, 130-137.

(27) Sneddon, I. N. The Relation between Load and Penetration in the Axisymmetric Boussinesq Problem for a Punch of Arbitrary Profile. Int. J. Eng. Sci. 1965, 3, 47-57.

(28) Langmuir, I. The Constitution and Fundamental Properties of Solids and Liquids. Pt I. Solids. J. Am. Chem. Soc. 1916, 38, 22212295. 
TOC figure

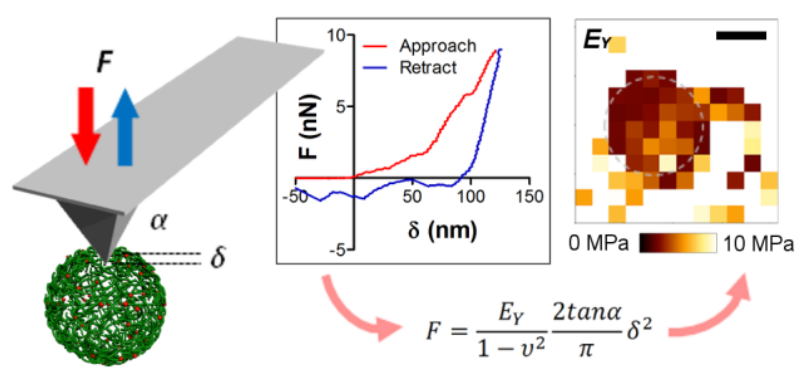

